\title{
NOUVELLE
}

\section{Des Casques Bleus chez les plantes Un microARN, miR164, stabilise les frontières dans le méristème}

Alexis Peaucelle, Patrick Laufs

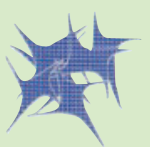

Laboratoire de Biologie Cellulaire, Institut Jean-Pierre Bourgin, Inra, 78026 Versailles Cedex, France. laufs@versailles.inra.fr
$>$ Chez les plantes, tous les organes latéraux de la partie aérienne, qu'il s'agisse de feuilles ou de pièces florales comme les pétales ou les étamines, sont issus des méristèmes. Ces structures en prolifération contiennent les cellules souches de la plante et sont le siège d'une organogenèse continue. Au sein des méristèmes, suivant un minutage et à une position finement contrôlés, un groupe de cellules va s'individualiser, bourgeonner en un primordium et donner naissance à un organe (Figure 1). Les primordia d'organes sont séparés entre eux et du méristème par un domaine frontière formé par un ruban de cellules. Les cellules appartenant à ces domaines sont reconnaissables par les gènes qu'elles expriment. Ainsi, chez la plante modèle Arabidospis thaliana, les cellules des domaines frontières expriment les gènes CUC1, 2 et 3 [1-3]. Ces marqueurs moléculaires des domaines frontières en sont

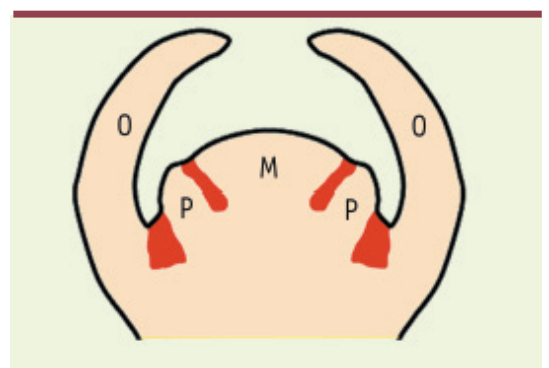

Figure 1. Méristème et domaines frontières. Le méristème $(M)$ produit des primordia $(P)$ sur ses flancs qui se développeront en organes (0), telles les feuilles. Un domaine frontière ici en rouge sépare les primordia entre eux et du méristème. aussi des déterminants majeurs, puisque l'inactivation d'un ou de plusieurs d'entre eux conduit à des défauts de spécification du domaine frontière entraînant des fusions entre organes. Par exemple, le double mutant cucl cuc2 se caractérise par une fusion des cotylédons, leur donnant une apparence de coupe (d'où le nom des gènes CUP-SHAPED COTY$\angle E D O N$, cotylédon en forme de coupe), et une fusion entre sépales ${ }^{1}$ et entre étamines ${ }^{2}$. Les gènes $C U C 1,2$ et 3 codent pour des protéines qui font partie de la famille des facteurs de transcription de type NAC, comprenant chez Arabidopsis

${ }^{1}$ Pièce du calice, soudée ou libre. Le sépale est une protection du bourgeon avant que la fleur n'éclose.

2 Étamine: partie mâle d'une fleur où le filet porte l'anthère (sac à pollen).

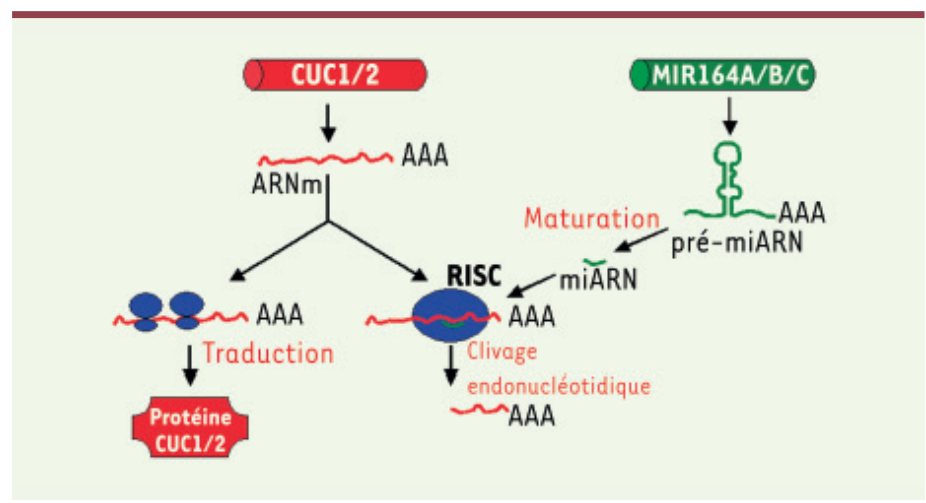

Figure 2. Régulation des gènes CUCl et 2 par miR164. Le microARN, miR164, est un inhibiteur de l'expression des gènes CUCl et 2. En l'absence de miR164, les gènes $C U C l$ et 2 sont transcrits en ARNm qui eux-mêmes sont traduits en protéines. La transcription des gènes MIRI64A, $B$ et $C$ produit des pré-miARN qui, après plusieurs étapes de maturation, donnent naissance au miARN, miR164. Ce dernier, au sein d'un complexe protéique appelé RISC (RNA-induced silencing complex), reconnaît les transcrits des gènes $C U C l$ et 2 et provoque leur clivage endonucléotidique. Les deux produits ainsi engendrés sont dégradés. plus d'une centaine de membres. Bien que très proches, ces trois gènes se distinguent néanmoins par l'existence d'un site de fixation d'un microARN (miARN), miR164, sur les transcrits des gènes CUC1 et CUC2, ce site étant absent du transcrit de CUC3.

Les miARN, identifiés à la fois chez les animaux et les végétaux, sont de petits ARN simple brin d'environ 21 nucléotides qui règlent de façon post-transcriptionnelle l'expression de gènes cibles avec lesquels ils présentent une complémentarité de séquence [4]. Chez les plantes, les miARN ont été identifiés physiquement par séquençage de banques de petits ARN ou prédits par différents algorithmes bio-informatiques. Parallèlement, les cibles potentielles de ces miARN ont été prédites grâce à la complémentarité de séquence entre le miARN et ses cibles. A i n s i, miR 164 pourrait cibler 6 facteurs de transcription de la famille NAC, dont CUCl et CUC2, et serait pro- 
duit à partir de 3 gènes MIRI64A, B et $C$ [5] (Figure 2).

Ces prédictions ont été validées expérimentalement de plusieurs façons. La plupart des miARN végétaux contrôlent l'expression de leurs cibles en induisant le clivage de leurs transcrits entre les nucléotides appariés aux $10^{\mathrm{e}}$ et $11^{\mathrm{e}}$ nucléotides du miARN. La détection des produits de clivage peut alors servir de trace de l'action du miARN. De tels produits de clivage ont été effectivement observés pour CUCI et CUC2 [6]. Le séquençage de l'extrémité du fragment 3' a révélé que le clivage avait eu lieu au niveau du site de fixation de miR164, impliquant ainsi directement ce miARN dans ce processus. De plus, miR164 est effectivement produit par les 3 gènes MIRI64A, $B$ et $C$, puisque leur surexpression provoque une augmentation du contenu en miR164, ce qui entraîne une diminution du niveau d'accumulation des transcrits des gènes CUCl et 2. Comme attendu, la réduction de l'expression des gènes CUC conduit à des anomalies développementales embryonnaires ou florales similaires à celles des doubles mutants cucl cuc2 [7-10] (Figure 3).

Se pose ensuite la question du rôle de la régulation des gènes CUC1 et CUC2 par miRl64 au cours du développement. Pour y répondre, une démarche classi-

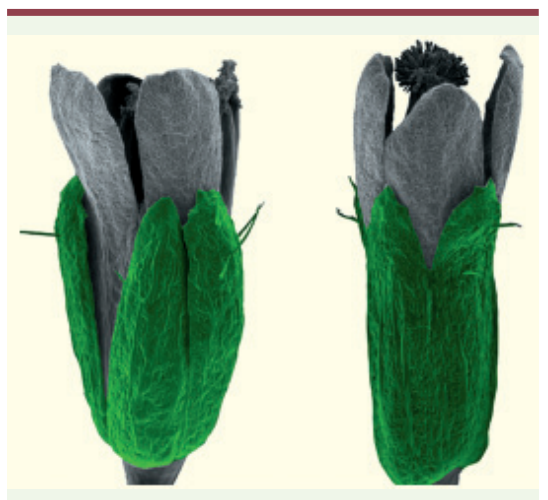

Figure 3. Modification de la morphologie florale provoquée par la surexpression de miR164. Chez des fleurs de plantes de type sauvage, les sépales (en vert) sont libres (fleur de gauche), alors que les fleurs de plantes surexprimant miRl64 (fleur de droite) développent des sépales fusionnés. que de génétique a été suivie, visant à perturber ou à bloquer cette régulation et à en analyser les conséquences. La première approche utilisée fut d'exprimer des versions de CUC1 ou CUC2 résistantes à l'action du miARN. Ces versions résistantes ont été créées en introduisant des mutations dans le gène cible au niveau du site de fixation du miARN, empêchant ainsi la formation de duplex miARN/cible. Pour ne pas modifier en même temps la fonctionnalité de la protéine, on a bien pris garde de n'introduire que des mutations silencieuses sans effet sur la séquence protéique. L'expression des formes résistantes à miRl64 de CUCl ou 2 perturbe le développement de la plante, alors que l'expression de formes non modifiées de ces gènes reste sans effet, soulignant l'importance de la régulation de ces deux gènes par miRl64. Plus précisément, les plantes exprimant une forme modifiée de CUCI produisent un nombre anormal d'organes floraux [7, 9]. De telles modifications du nombre d'organes ne sont pas observées chez des plantes exprimant une version miRl64résistante de CUC2, qui montrent quant à elles un élargissement du domaine frontière [8]. Cet élargissement est visible morphologiquement, en particulier au niveau des sépales. II est aussi directement visible au niveau cytologique au cours du développement de la fleur grâce à l'expression d'une protéine fluorescente, la GFP, dans les cellules faisant partie du domaine frontière. Un tel élargissement des frontières est également observé chez des mutants affectés dans la biogenèse des miARN et qui présentent une accumulation réduite de miR164 [8]. L'analyse de ces mutants, bien que confirmant le rôle de miR164 dans la stabilisation des frontières, reste délicate car un grand nombre, sinon la totalité, des miARN y sont modifiés, conduisant à une large gamme de perturbations. L'alternative est d'analyser des mutants affectés spécifiquement dans les gènes MIRl64. La première mutation décrite inactive le gène $M I R 164 B$ mais, bien que réduisant fortement la teneur en miR164, elle ne conduit à aucun défaut décelable dans le fonctionnement du méristème, suggérant que les deux autres gènes MIRI64A et $C$ toujours fonctionnels sont suffisants [7]. Au contraire, l'inactivation partielle du gène MIRI64C conduit à une augmentation du nombre de pétales comme cela avait déjà été observé chez des plantes exprimant une forme miRl64-résistante de CUCl [9]. Ces modifications sont dues à une expression plus forte des gènes CUC1 et CUC2 et à une fragmentation de leur domaine d'expression, ce qui serait la cause de la formation des organes surnuméraires. Néanmoins, dans ce mutant, le domaine d'expression des gènes CUC n'est pas élargi, ce qui suggère que MIRI64C ne limiterait pas l'expansion du domaine frontière mais contrôlerait le niveau d'accumulation des transcrits CUC au sein de ce domaine. Cette hypothèse est renforcée par l'observation que MIRI64C est exprimé dans un domaine chevauchant celui des gènes CUC.

L'ensemble de ces observations suggère deux mécanismes par lesquels miR164 contribue à stabiliser les frontières. Le premier, révélé par le phénotype des plantes exprimant une version miR164résistante de CUC2, est de limiter l'expansion des frontières. Le second, mis en lumière par le mutant mirl64c, est de tamponner l'expression des gènes CUCl et 2 au sein même des domaines frontières. Des questions subsistent néanmoins: quel est le rôle des gènes MIRI64A et $B$ ? $\varepsilon t$ quelles seraient les conséquences sur la plante d'une absence totale de miRl64?

L'analyse du rôle de miRl64 a mis en évidence un nouveau niveau de régulation du développement des domaines frontières dans le méristème et illustre le rôle des miARN dans la régulation de gènes-clés du développement. Dans le cas de miR164, la régulation est probablement très ancienne, puisque des homologues des gènes CUCI et 2 possédant un site de fixation de miRl64 
et miRl64 lui-même sont présents chez d'autres dicotylédones, des monocotylédones et même des gymnospermes ${ }^{3}$ $[7,11]$. Cette conservation et le degré de redondance génique laissent penser

${ }^{3}$ Les plantes à fleurs, ou angiospermes, groupe immense de plus de 250000 espèces, se distinguent des autres végétaux car elles portent, durant une partie de leur cycle, des organes sexuels mâles et femelles généralement rassemblés en une fleur bisexuée. L'ovaire, partie femelle de la fleur, est constitué d'ovules entourés d'écailles ou carpelles (du grec karpos $=$ fruit). Après la fécondation, les ovules se transforment en graines, l'ovaire se transforme en fruit. Les angiospermes comprennent deux classes, les monocotylédones et les dicotylédones. À la germination de la graine, l'embryon possède des cotylédons, sortes de «feuilles» embryonnaires qui lui servent de réserve. Chez les monocotylédones, un des deux cotylédons ne se développe pas, reste dans la graine ou dans le sol: seul l'autre émerge. Les gymnospermes (littéralement «graines nues») sont des plantes dont les ovules libres (non enclos dans un ovaire à la différence des angiospermes) sont fécondés directement par le pollen. Les conifères sont les principaux représentants des gymnospermes dans notre flore actuelle (source: http://www.jeanlouisetienne.fr/clipperton et www.botanique.org). que les domaines frontières pourraient avoir un rôle plus important que la seule séparation des organes. Un tel rôle reste toutefois à découvrir. $\diamond$

The UN peace-keeping force in the plants: a microRNA, miR164, stabilizes the boundary domain in the meristem

\section{RéFÉRENCES}

1. Takada S, Hibara K, Ishida T, et al. The CUP-SHAPED COTYLEDONI gene of Arabidopsis regulates shoot apical meristem formation. Development 2001 ; $128: 1127-35$.

2. Aida M, Ishida T, Fukaki $H$, et al. Genes involved in organ separation in Arabidopsis: an analysis of the cup-shaped cotyledon mutant. Plant Cell 1997 ; 9: 841-57

3. Vroemen CW, Mordhorst AP, Albrecht C, et al. The CUPSHAPED COTYLEDON3 gene is required for boundary and shoot meristem formation in Arabidopsis. Plant Cell $2003 ; 15: 1563-77$.
4. Bartel DP. MicroRNAs. Genomics, biogenesis, mechanism, and function. Cell $2004 ; 116: 281-97$.

5. Rhoades MW, Reinhart BJ, Lim LP, et al. Prediction of plant microRNA targets. Cell $2002 ; 110: 513-20$.

6. Kasschau KD, Xie Z, Allen E, et al. Pl/HC-Pro, a viral suppressor of RNA silencing, interferes with Arabidopsis development and miRNA function. Dev Cell 2003; 4 : 205-17.

7. Mallory AC, Dugas DV, Bartel DP, et al. MicroRNA regulation of NAC-domain targets is required for proper formation and separation of adjacent embryonic, vegetative, and floral organs. Curr Biol $2004 ; 14: 1035-46$.

8. Laufs P, Peaucelle A, Morin H, et al. MicroRNA regulation of the CUC genes is required for boundary size control in Arabidopsis meristems. Development 2004 ; 131 : 4311-22.

9. Baker CC, Sieber P, Wellmer F, et al. The early extra petalsl mutant uncovers a role for microRNA miR164c in regulating petal number in Arabidopsis. Curr Biol $2005 ; 15: 303-15$.

10. Schwab R, Palatnik JF, Riester M, et al. Specific effects of microRNAs on the plant transcriptome. Dev Cell $2005 ; 8: 517-27$.

11. Axtell MJ, Bartel DP. Antiquity of microRNAs and their targets in land plants. Plant Cell $2005 ; 17$ : 1658-73.
NOUVELLE

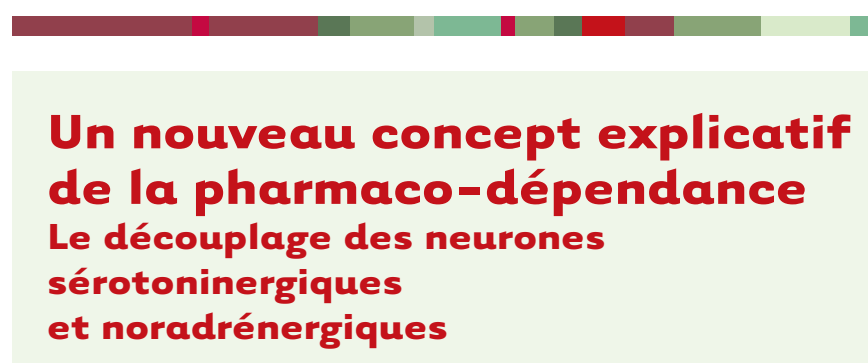

Jean-Pol Tassin, Christophe Lanteri, Lucas Salomon

$>$ Le cerveau constitue un ensemble complexe de circuits neuronaux qui s'organisent en réseaux pour traiter les entrées sensorielles, les relayer jusqu'au cortex, puis les traduire en sorties comportementales ou psychiques. La grande variété des réponses comportementales nécessite que certains réseaux, et par conséquent certaines structures cérébrales, soient sélectionnés en fonction de chaque situation vécue par le sujet. Cette sélection est réalisée par un autre ensemble de neurones, modulateurs, superposé au premier circuit. Ces neurones modulateurs, minoritaires dans le système nerveux central puisqu'ils représentent moins de un pour cent des cent milliards de cellules présentes dans le cerveau, comprennent les neurones sérotoninergiques, noradrénergiques et dopaminergiques. Dans cet ensemble, la dopamine tiendrait le rôle de modulateur final de l'essentiel des sorties motrices ou psychiques. C'est pourquoi une atteinte du système dopaminergique peut se traduire aussi bien par des troubles moteurs, comme dans le cas de la maladie de Parkinson, que psychiques, comme dans certaines psychoses telle que la schizophrénie.

Dans les mécanismes de dépendance, le système dopaminergique est aussi déterminant dans la mesure où il modifie le fonctionnement d'un ensemble neuronal particulier, le «circuit de la récompense » qui relaie toutes les infor-
Inserm U114, CNRS UMR 7148,

Collège de France,

11, place Marcelin Berthelot,

75005 Paris, France.

jean-pol.tassin@college-de-france.fr

mations externes et internes de l'organisme et permet au sujet de reconnaître, par l'intermédiaire de perceptions extérieures, l'existence de satisfactions potentielles de toutes sortes: nourriture, chaleur, plaisir sexuel... Ce circuit de la récompense est, en quelque sorte, un «baromètre » qui indique à l'individu l'état physique et psychique dans lequel il se trouve ou va se trouver. Les neurones dopaminergiques ne font pas partie à proprement parler du circuit de la récompense, mais leur activation stimule ce circuit et provoque une sensation de satisfaction. 